REVISTA PROYECCIONES $\mathrm{N}^{\circ} 9$ : 133-177

Julio 1985 - I.S.S.N. 0716-0917.

\title{
EL PROGRAMA STAT 11
}

PATRICIO ROJAS MORERA :

En la actualidad existe una gran cantidad de programas iteractivos; todos tienen virtudes y defectos, no por su construcción, sino desde el pun to de vista del usuario, de lo que el usuario espera del programa, en este caso se analiza el programa Stat 11 , disponible en el Centro de Computación de 1a Universidad del Norte; que reside en el disco de usuarios del equipo VAX II/750 y que es accesible por medio de terminales del tipo VT 101 VT 102 .

Este programa es útil para investigadores que usan la estadística como una herramienta básica para sus investigaciones, en el sentido que per mite hacer una gran cantidad de análisis, ya sea del tipo paramétrico, o no paramétrico, como también análisis descriptivos. Una de sus grandes virtu-

* DEPARTAMENTO DE MATEMATICAS, FACULTAD DE CIENCIAS, UNIVERSIDAD DEL NORTE. 
des es su potencialidad en gráficos de distintos tipos. La utilidad de este trabajo radica en que se ha ordenado en forma muy simple las distintas secuencias de comandos para ingresar datos como también para realizar los diferentes análisis.

Este programa puede trabajar con una matriz de datos definida al iniciar cada análisis, como también con submatrices, que se obtienen al eliminar columnas; tambiên permite cambiar la variable de respuesta en forma muy sencilla, lo que hace que el programa sea muy dinámico, que es 10 que generalmente interesa al investigador, probar, o como se dice a veces "jugar" un poco con los datos, $\mathrm{L}$ "ando las relaciones entre las variables en estudio.

Todo 10 anterior unido a una gran rapidez de respuesta que puede ser por terminal o por impresora hace que el STAT 11 sea recomendable para quienes deseen hacer análisis estadísticos en sus investigaciones.

La idea original al comenzar este trabajo era explica cada análisis desde su concepción teórica hasta el análisis de resultados, en los formatos que entrega el STATT 11, algunos de los cuales no son muy explicativos, y que pueden ser cambiados; para concretar esta idea original sig nificaría triplicar el volumen del anexo en que se encuentra explicado el programa STAT 11 .

E1 programa STAT 11, además del inğreso de datos, puede realizar las siguientes funciones, entre otras:

1. Listado de datos.

2. Cambio de matriz de datos.

3. Câlculo de medias y desviaciones típicas

4. Prueba $t$.

5. Recta de regresión.

6. Recta de regresión de Bartletts.

7. Matriz de correlación.

8. Anova de 1 criterio. 
9. Anova a 2 criterios.

10. Medias y desviaciones de problaciones logarítmicas.

11. Prueba chi-cuadrado.

12. Prueba de la mediana.

13. Prueba de Wilcoxon

14. Prueba de Mann-Whitney.

15. Prueba de Kruskall-Wallis.

16. Prueba de Spearman

17. Anova de Friedman

18. Integración numérica.

19. Tabulación cruzada.

20. Gráficos.

21. Conversión de columnas.

22. Regresión múltiple.

23. Anoval multicriterio.

24. Regresión polinómica.

25. Regresión múltiple (Step wise).

26. Prueba de Newman-Keul.

En el Anexo titulado "Manual de Usuario STAT 11", se puede encontrar la forma de trabajar con el STAT 11. 


\section{MANUAL DE USUARIO STAT 11.}

En este trabajo se describe las potencialidades del paquete STAT 11 disponible en el disco de usuarios del CECUN (Centro de Computación de la Universidad del Norte), del equipo VAX II/750 y accesible por terminal del tipo VT101 - VT102.

Se ha tratado de ser lo más explicativo posible en cuanto a la forma de usar este paquete, el deseo iniclal del autor era explicar cada análisis en forma detallada en cuanto a su concepción teórica, incluyendo las explicaciones de los resultados, pero este trabajo demandaría una publicación que triplicaría a ésta, por lo que se ha dejado esta idea para un próximo trabajo.

Para conectarse al paquete STAT 11, digite STAT 11, al hacerlo aparece la siguiente pantalla:

MENU STAT-11

(1) Documentación del paquete STAT

(2) Ejemplo entrada de datos

(3) Opciones para la entrada de datos

*(4) ENTRADA DE DATOS (OUTLAY)

(5) Ejemplo análisis de datos

(6) Opciones para el análisis de datos

(7) Análisis estadísticos posibles

*(8) ANALISIS (STAT)

(9) Datos desde un editor (OUTCHK)

(10) Análisis especiales (TABELL)

(99) Fin

OPCION : 
Este es el meru principal de STAT 11 , en ella aparecen 11 opcio nes, que revisaremos socuencialmente.

1. (1) DOCUMENIACION DEL PAQUETE STAT.

Al digitar l aparece la signiente pantalla

OPCION: DOCLMENTACION DEL PAQUETE STAT

Proporcionada por:

- Ma inel del usuario

- H. de sistema

- Ht interactivo

Indique opciön:

(1) Marual de Usuario

(2) Help de sistema

(3) Help interactivo

(99) Fin documentación

DPCION :

FANTELLA 2

1.1. (1) Manual de Usuazio.

For el morento esta opciśn no está implementada, sin embargo puede ser solicitada a Univergidad del. Norte, CECUN. Casilla 1280 - An tofagasta.

Ai digitar l, Sir vuelve a la pantalla 2.

1.2. (2) Helf del sistema.

Por el momento esta opción tampoco está implementada.

Al digitar 2 aparece en pantalla el listado del Help del sistema VAX, a la pregunta Topic? que aparece, digite <RETURN>, aparece nuevamente la pantalia 2 . 
1.3. (3) Help Interactivo.

Al digitar $\underline{3}$, STAT despliega la siguiente pantalla:

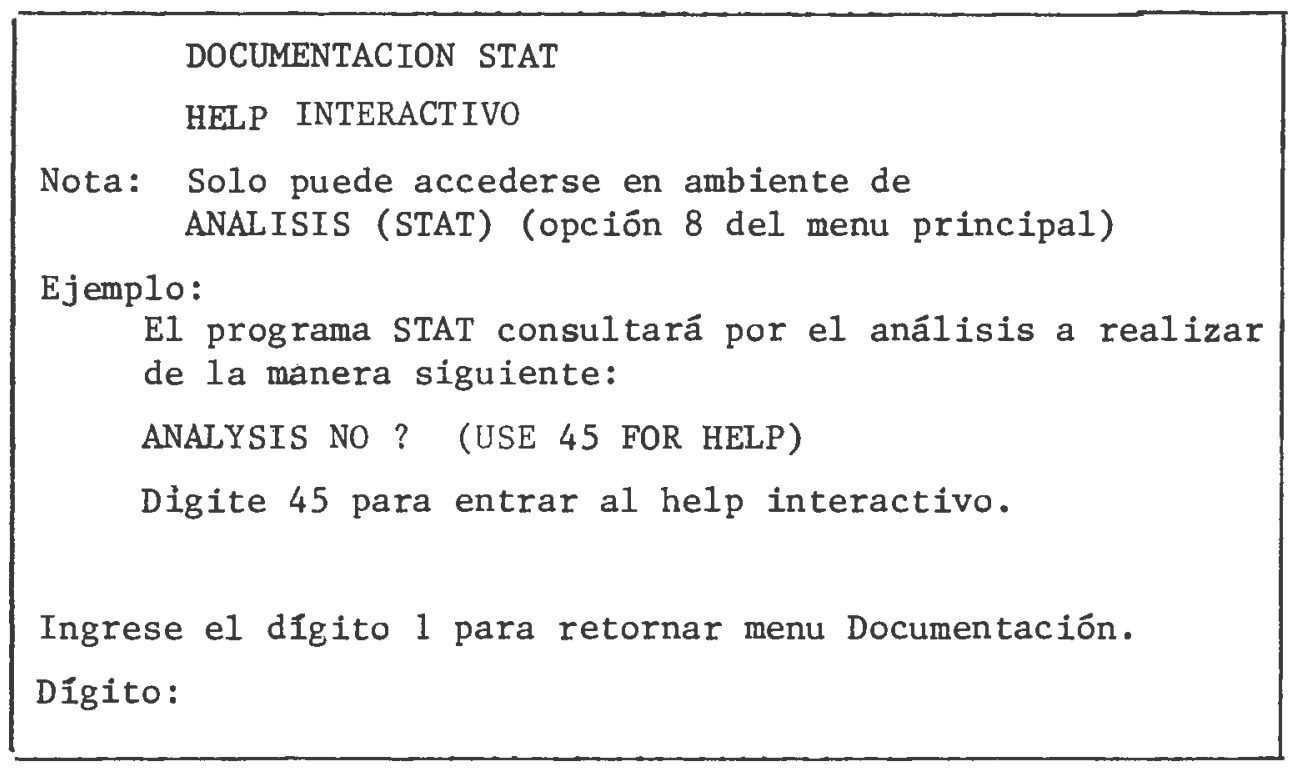

PANTALLA 3

en el cual se indica como usar el Help Interactivo. Al digitar 1 después de esta pantalla, STAT vuelve a la pantalla 2.

1.4. (99) Fin Documentación.

Al digitar 99 , STAT vuelve a la pantalla 1. 
2. (2) EJEMPLO ENTRADA DE DATOS.

Al digitar 2 en la pantalla 1, STAT despliega la siguiente pantalla:

OPCION: EJEMPLO ENTRADA DE DATOS

Nota: Se debe estar en ambiente ENTRADA DE DATOS (OUTLAY) (opción 4 del menu principal).

Dependiendo de las opciones que Ud. indicó para

la entrada de datos, el sistema consulta por:

+ código para Missing values

+ identificación (heading) del trabajo

+ tipo de formato a usar para el archivo de salida en el caso de indicar formato no outlay.

+ archivo de entrada de datos a anexar al que se crea

+ heading para los grupos

Además pregunta por:

+ número de grupos en que se ha dividido el trabajo (división de la muestra en submuestras)

+ número de columnas para el trabajo (las columnas representan las variables en estudio para la muestra)

Presione $<\mathrm{CR}>$ para continuar:

SIGUE : 
Al presionar <RETURN> después de esta pantalla, STAT despliega

la siguiente:

Si se tiene la siguiente información de peso, edad, estatura (variables= peso, edad, estatura), para hombres y mujeres (grupos= hombres, mujeres), y se desea hacer análisis estadisticos tanto de cada uno de los grupos por separado como de todos los grupos en forma conjunta, la manera de entrar los datos al archivo es:

a la consulta GROUPS? ingresar 2

COLUMNS? ingresar 3

ingresar los datos del grupo uno (hombres)

Variable1, variable2, variable

n $11 " 11011$

" 11 " 11

CTRL/2 (fin de datos)

ingresar los datos del grupo dos (mujeres)

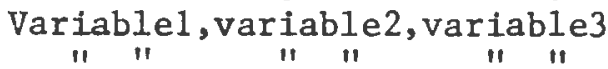

" 11 " 11 "

CTRL/2" ( in de datos)

1111

Presione $<\mathrm{CR}>$ para continuar!

SIGUE:

PANTALLA 5 
Al presionar <RETURN> después de esta pantalla, STAT despliega la siguiente:

IMPORTANTE: Si desea heading para las columnas (variables) debe crear mediante un editor un archivo con nombres de columnas, o mediante el análisis 44 cuando está en el ambiente ANALISIS (STAT). En el primer caso los nombres de las columnas son permanentes para toda la corrida del programa y en el segundo son nombres temporales (designación dura hasta un análisis 50 o 0 de STAT). Si se tiene el archivo de headings de columnas y se está en ambiente ANALISIS (STAT) so debe colocar la opción /c y además dar el nombre l's l. archivo de headings.

En el ejemplo anterior se puede tener un archivo de nombres de columnas 1 lamado col.dat que contiene 1o siguiente:

$$
\begin{aligned}
\text { top-file } \rightarrow & \text { peso } \\
& \text { edad } \\
& \text { estatura } \leftarrow \text { bottom-file }
\end{aligned}
$$

Digite un 1 para volver al menu principal

DIGITO? :

\section{PANTALLA 6}

Las pantallas 4, 5 y 6 explican el uso de la opción entrada de datos, este procedimiento se explica más adelante. Al digitar 1 después de esta pantalla, STAT vuelve a la pantalla 1. 
3. (3) OPCIONES PARA LA ENTRADA DE DATOS.

Al digitar 3 en la pantalla 1, STAT despliega la siguiente panta 11a:

OPCION: OPCIONES PARA LA ENTRADA DE DATOS

Nota: Las opciones son ingresadas de la siguiente manera:

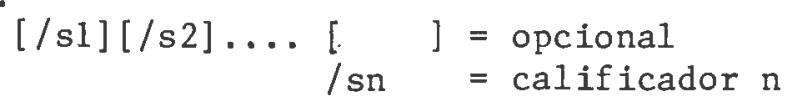

Si no desea ingresar opciones presiones <CR> Con esto, asume:

$$
\begin{aligned}
& \text { - código para missing es } 0 \\
& \text { - entrada solo por teclado } \\
& \text { - se crea archivo salida con } \\
& \text { formato outlay. }
\end{aligned}
$$

Calificadores:

$$
\begin{aligned}
& \text { /c :Consulta código para missing values } \\
& / \mathrm{h}: \text { :Consulta headings para cada grupo } \\
& \text { /a : " " archivo datos de entrada } \\
& \text { /q :El archivo de salida no tiene formato outlay } \\
& / \mathrm{f}: \text { Consulta por un formato de datos no outlay }
\end{aligned}
$$

Digite 1 para retornar al menu principal

DIGITO? :

\section{PANTALLA 7}

Esta pantalla explica el uso de calificadores y valores que asume por defecto. Al digitar 1 después de esta pantalla STAT vuelve a la pantalla 1 . 
4. *(4) ENTRADA DE DATOS (OUTLAY).

A1 digitar 4, STAT despliega la siguiente pantalla:

\section{OPCION: ENTRADA DE DATOS (OUTLAY)}

Inmediatamente después que en su pantalla tenga un párrafo de 4 líneas con información no relevante, Ud. opcionalmente puede indicar lo siguiente:

+ lista de opciones para la entrada de datos o $\langle\mathrm{CR}\rangle$ (Ver opción 3 del menú principa1)

+ nombre del archivo de salida (debe ser con extensión .dat) o $\langle\mathrm{CR}\rangle$ con 10 que asume que el archivo de salida será el for007.dat

Digite 1 para retornar al menu principal $\circ\langle\mathrm{CR}\rangle$ para continuar

PANTALLA 8

Esta pantalla indica los pasos a seguir después de digitar 4 en la pantalla 1. Al digitar 1 después de esta pantalla, STAT vuelve a $1 \mathrm{a}$ pantalla 1. Al digitar <RETURN> STAT despliega las siguientes 4 líneas que indica la pantalla 8

OUTLAY: VAX VERSION G.51- 10000. ON 1985-3-14 NOTE !!

$\operatorname{YES}(\mathrm{JA})=1(>1)$

NO $($ NEJ $)=0 .($ BLANK LINE)

Después de estas 4 líneas STAT queda esperando un string de calificadores que son los indicados en la pantalla 7 (ver apartado 4.1) en este momento, se puede digitar el string de caracteres, o poner <RETURN> con 1o que STAT asume los valores por defecto indicados también en la pantalla 7, después de cualquiera de las opciones anteriores (string de calificadores o (RETURN>), STAT queda esperando el nombre del archivo de salida que debe ser del $x x x x x x x x x x x$.DAT, donde las $x$ 's indican el máximo de caracteres alfanuméricos. 
Después de poner el nombre del archivo de salida o <RETURN> (con 1o que asume que el archivo de salida tendrá el nombre de FOR007.DAT), STAT consulta por el número de grupos (NO OF GROUPS), según se explica en la pantalla 5, después de digitar el número de grupos, STAT consulta por el número de colum: número de columnas STAT consulta por el título que identifica los datos (MAIN HEADING:) que puede ser de cualquier extensión, después de digitar el MAIN HEADING, aparecen las siguientes dos líneas WAIT

INPUT X-VALUES FOR GROUP 1 AND FINISH WITH EOF く^Z> Y STAT consulta por los datos del primer grupo que deben ser ingresados por filas y separados por comas (cada quien debe contener tantas columnas como se haya indicado en la consulta NO OF COLUMNS). Después de terminado el ingreso de datos del grupo 1, se digitan las teclas CTRL y z simultánea mente, con lo cual se indica a STAT que ha terminado de ingresar el primer grupo. Después de esta -operación ( $\langle\mathrm{Z}\rangle)$, si a la consulta No OF GROUPS, se ha contestado con un dígito mayor que 1, STAT despliega las siguientes líneas:

INPUT DATA END

$\mathrm{N}$ POWS TOTAL N ROWS IN BUFFER

INPUT X-VALUES FOR GROUP 2 AND FINISH WITH EOF <`z> donde $\mathrm{N}$ indica el número de filas que Ud. ingresó en el grupo anterior. Ahora STAT espera el ingreso de datos para el grupo siguiente.

Después de ingresar el último grupo de datos STAT despliega las siguientes líneas

INPUT DATA END

$\mathrm{N}$ ROWS TOTAL M ROWS IN BUFFER

LIST? <0/1>

donde $\mathrm{N}$ indica el total de filas ingresadas y $\mathrm{M}$ el total de filas ingresadas en el último grupo. A la consulta LIST? $<0 / 1>$, poner 0 indica que no desea listado de los datos por pantalla, poner 1 indica que se desea un listado por pantalla que es presentado de la siguiente forma: RON GROUP $\mathrm{N}_{1} \mathrm{~N}_{2} \mathrm{~N}_{3} \cdots \cdots$ 
donde ROW indica la fila, GROUP el grupo y $\mathrm{N}_{1}, \mathrm{~N}_{2}, \mathrm{~N}_{3}, \ldots$ los datos de la fila. En cualquiera de los dos casos ( 0 ó 1) STAT consulta si se ha cometido errores en la entrada de datos de la siguiente forma:

ERRORS?

ROW, COL, VALUES... <END= ROW O>

Aquí se pueden arreglar los errores cometidos al digitar los datos, para lo cual debe colocarse un string de números en el orden ROW, COL, VALUES es decir la fila y la columna en que se ha comedito el error y luego el valor correcto, todo separado por comas. Después de corregir todos los posibles errores, se pone un cero al iniciar la líneas y nuevamente STAT despliega la lineas LIST? $<0 / 1>$ si se responde con cero, consulta por ERRORS? y si se vuelve a digitar cero, STAT ruelve a la pantalla 1 y crea el archivo de datos con el nombre que Ud. ha ingresado previamente o le asigna el nombre FORDD7.DAT si Ud. no ha ingresado el nombre del archivo de datos.

\subsection{Uso de los calificadores en la opción 4.}

Los calificadores que se pueden usar son los indicados en la pan talla 7 , es decir

$$
\begin{aligned}
& \text { /C: código para datos missing } \\
& \text { /H: títulos para cada grupo } \\
& \text { /A: archivo de datos de entrada } \\
& \text { /Q: formato del archivo de salida } \\
& \text { /F: entrada de datos con formato especial }
\end{aligned}
$$

4.1.1. Uso de /C (código para datos missing)

Al usar /C, STAT consulta por

NO OF GROUPS

NO OF COLUMNS

MAIN HEADING

que se responden según lo que se ha explicado anteriormente. 
A la consulta siguientes que es MISSING CODE?

se debe responder con un valor numérico que STAT considerará como valor missing y cada vez que lo encuentre en el set de datos lo indicará como la palabra MISSING o con * $*$ (asteríscos), según el análisis que se esté realizando. E1 valor por defecto (cuando no se usa la opción/C) es cero $(0.00)$.

4.1.2. Uso de /H (títulos para cada grupo)

Al usar /H, STAT consulta

NO OF GROUPS

NO OF COLUMNS

MAIN HEADING

que se responden según lo explicado antes. A la consulta siguiente que es:

N GROUP HEADING ON SEPARATE LINES

se debe responder con un string de caracteres alfanuméricos para cada grupo de datos (un título en cada línea), el efecto es que cada grupo de datos queda bajo un título, además del título general del set de datos (MAIN HEADING) .

4.1.3. Uso de /A (archivos de datos de entrada)

Para usar este calificador se debe tener un archivo de datos. Al usar /A, aparecen las siguientes dos líneas

HEADING \& GROUPS FROM UNIT NR

$0=$ AUTO $\quad 4=$ TERMINAL $8-9=$ FILE

STAT nos presenta tres alternativas que analizaremos secuencialmente.

4.1.3.1. Uso de $0=$ AUTO en la línea (1)

Al digitar la opción 0 (cero), permite elegir algunos grupos y algunas columnas del archivo de datos original y si se quiere cambiar el 
título de los datos, para lo cual STAT hace las consultas

NO OF GROUPS

NO OF COLUMNS

MAIN HEADING

Después de responder a estas consultas STAT, despliega las dos lineas sí guientes

WAIT

INPUT FILE UNIT NO; MUST BE 8 TO 9 ; <0 IF NON>?

con 10 cual consulta si existe o no un archivo de datos.

4.1.3.1.1. Uso de $\emptyset$ en la línea (2)

Al digitar 0 en la línea (2) se está indicando que no existe un archivo de datos. Después de digitar 0 STAT despliega las siguientes dos lineas

\section{NEW INPUT?}

[TTY=4; OTHERS STATE UNIT NR <8 TO 9]

con 10 cual consulta si desea agregar nuevos datos ya sea por terminal o por archivos.

4.1.3.1.1.1. Uso de <RETURN> en la línea (3)

Al digitar <RETURN> en la línea (3) se está indicando que no se ingresarán nuevos datos, después de digitar <RETURN> en 1a 1ínea (3), STAT despliega la siguiente línea

$$
\text { O.K. TO CLOSE FILE WITH NO OUTPUT? }
$$

a la cual se debe responder obligadamente YES, con lo cual STAT vuelve a la pantalla 1. Cualquier otra respuesta distinta de YES, produce un error.

4.1.3.1.1.2. Uso de 4 en la linea (3)

Al digitar 4 en la línea (3) se está indicando que se ingresa rán nuevos datos por terminal. Al digitar 4 en la línea (3) STAT desplie ga la siguiente línea 
ENTER GROUP NR AND X VALUES END WITH BLANK LINES

con la cual pide que se ingresen los datos, los que se deben ingresar por filas separados por comas y el primer dígito debe corresponder al grupo al cual pertenece la fila de datos. Se termina el ingreso de datos con una línea en blanco, después de lo cual STAT despliega las siguientes 1íneas

INPUT DAT END

$\mathrm{N}$ ROWS TOTAL N ROWS IN BUFFER

LIST? $<0 / 1>$

y se sigue el procedimiento indicado en el apartado 4, con la diferencia que al digitar 0 en la línea

ROW, COL, VALUES. . . <END=ROW O>

STAT despliega las siguientes líneas

WAIT

NEW INPUT?

ONLY TTY (UNIT 4) ALLOWED

con 10 cual consulta si desea ingresar nuevos datos.

4.1.3.1.1.2.1. Uso de 〈RETURN> en la linea (7)

Al digitar <RETURN> en la línea (7) se está indicando que no hay nuevos datos, con lo cual STAT despliega la pantalla 1 y cierra el archivo de datos.

4.1.3.1.1.2.2. Uso de 4 en la linea (7)

Al digitar 4 en la línea (7) se está indicando que se digitarán nuevos datos por terminal. Al digitar 4 en la linea (7) STAT despliega la línea (5) y se sigue el procedimiento indicado en el apartado 4.1.3.1.1.2.

4.1.3.1.1.3. Uso de los números 7,8 ó 9 en la línea (3)

Al digitar los números 7,8 б 9 (todos producen el mismo efecto) en la línea (3) se está indicando que se ingresarán nuevos datos por archivos. Después de digitar algunos de estos números, STAT espera por el 
nombre del archivo, una vez ingresado este y si el formato de los datos es correcto, STAT los agrega a la cola del archivo de datos que se está generando y después despliega la línea (6) y se sigue el procedimiento indicado en el apartado 4, con la diferencia que al responder con 0 a 1 a línea ROW, COL, VALUES... <END-ROW O> STAT despliega la línea 3 y se sigue el procedimiento indicado en este apartado y en los apartados 4.1.3.1.1.1. y 4.1.3.1.1.2.

\subsection{Uso de 8 ó 9 en la línea (2)}

Al digitar los números 8 ó 9 (ambos producen el mismo efecto)en la línea (2) se está indicando que los datos están en un archivo, después de digitar 8 ó 9 STAT queda esperando el nombre del archivo (el archivo debe estar en el formato OUTLAY que usa STAT 11, cualquier otro formato provocará un error en la lectura). Después de digitar el nombre del archivo y si el formato de los datos está correcto, STAT despliega la línea (6) y se sigue el procedimiento indicado en el apartado 4, con la di ferencia que al digitar 0 en la línea ROW,COL, VALUES ... < END=ROW 0 ; STAT despliega la línea (2) y se sigue el procedimiento indicado en los apartados 4.1 .3 .1 .1 . y 4.1 .3 .1 .2 .

\subsubsection{Uso de 4 en la línea (1)}

Al digitar 4 en la línea (1) se está indicando esencialmente lo mismo que con la opción 0 , pero además STAT consulta por los títulos de cada grupo con la línea

$$
\text { N GROUP HEADING ON SEPARATE LINES }
$$

Después de ingresar los títulos de cada grupo, STAT despliega la línea (2) y se sigue el procedimiento indicado en los apartados 4.1.3.1.1. y 4.1 .3 .1 .2 .

4.1.3.3. Uso de 8 ó 9 en la línea (1)

Al digitar 8 ó 9 en la lînea (1) se está indicando que los datos están en un archivo de datos (en este caso se copian los datos tal 
como aparecen en el archivo). Después de digitar 8 o 9 STAT queda esperando que se ingrese el nombre del archivo, una vez ingresado éste, STAT despliega la línea (2), a la cual se debe responder con 8 ó 9 (según 10 que se haya digitado en la línea (1), después de ésto STAT despliega la línea (6) y se sigue el procedimiento indicado en el apartado 4, con 1a diferencia que al digitar 0 en la linea

$$
\text { ROW, COL, VALUES ... < END=ROW O> }
$$

STAT despliega la línea (2) v se sigue el procedimiento indicado en los apartados 4.1.3.1.1. y 4.1.3.1.2.

4.1.4. Uso de /Q (formato de archivo de salida)

Al usar /Q STAT consulta sólo por el número de columnas (NO OF COLUMNS), es decir bajo este calificador no se puede trabajar con grupos, después de ingresar el número de columnas, STAT despliega la línea INPUT X-VALUES FOR GROUP 1 AND FINISH WITH EOF $\langle 1 Z\rangle$ con lo cual pide que se ingresen los datos por filas y separados por comas, se termina el set de datos con $\langle$ CTRL $>Z$ y STAT despliega la línea (6) y se sigue el procedimiento indicado en el apartado 4, con la diferencia que al digitar 0 en la fila ROW, COL, VALUES... <END=ROW $0>$ STAT despliega la pantalla 1.

4.1.5. Uso de /F (entrada de datos con formato especial)

Este calificador hace 10 mismo que /A

5. (5) EJEMPLO DE ANALISIS DE DATOS.

Al digitar 5 en la pantalla l STAT despliega las dos pantallas siguientes: 
OPCION: EJEMPLO ANALISIS DE DATOS

Nota: Se debe estar en ambiente ANALISIS (STAT)

(Opción 8 del menu principa1)

Ejemplo: E1 programa STAT consultará por el analisis

a realizar de la manera siguiente:

ANALYSIS NO ? (USE 45 FOR HELP)

Ud. debe digitar un string de la forma:

$\mathrm{T}, \mathrm{A} 1, \mathrm{~A} 2, \mathrm{~A} 3, \ldots$.

En que $T$ es un dígito que indica el análisis

a realizar (Ver help interactivo o manual de usuario)

$A 1, A 2, A 3 \ldots$ son dígitos cuyo significado varía de un análisis a otro (Ver help interactivo o manual de usuario).

Presiones $<\mathrm{CR}>$ para continuar

SIGUE :

PANTALLA 9

Si se indica:

0 se desea considerar otro grupo y otras columnas para efectuar los análisis

1 se pide la media y el error para los grupos y columnas consideradas

5,A1 se pide análisis de correlación

22,A1,A2 pide transformaciones temporales de los datos

Digite 1 para volver al menu principal

DIGITO? :

PANTALLA 10

Al digitar 1 en la pantalla 10 STAT despliega la pantalla 1. 
6. (6) OPCIONES PARA EL ANALISIS DE DATOS.

Al digitar 6 en la pantalla 1 se despliega la siguiente panta11a:

OPCION: OPCIONES PARA EL ANALISIS DE DATOS

Nota: Las opciones son ingresadas como se indica:

[/SI][/S2]...[//Sn] [ ] = opcional

$/ \mathrm{Sn}=$ calificador $\mathrm{n}$

Si desea no ingresar opciones presione $\langle C R\rangle$, con 10 que asume salida por terminal y los análisis a efectuar se darán por teclado.

Calificadores:

/L salida por impresora

/n si se tiene además /L, inhibe salida por impresora y el resultado de los análisis se guarda en archivo creado por STAT.

$/ \mathrm{p}$ no paginación

$/ \mathrm{k}$ los análisis a efectuar se dan por archivo

/c los headings de columna se dan por archivo

/q el archivo de datos no tiene formato Outlay

Obs: Una lista completa de los calificadores se encuentra en el Help del sistema.

Digite 1 para retornar al menu principal

DIGITO?:

PANTALLA 11

Al digitar 1 en la pantalla 11 STAT despliega la pantalla 1. 
7. (7) PRINCIPALES ANALISIS DE STAT.

Al digitar 7 en la pantalla 1, STAT despliega la siguiente panta11a:

OPCION: Principales análisis de STAT

ANALISIS

Media y error standar

Media geométrica

$T$ test

Rēgresion 1 inea 1

Regresión de bartlette

Regresión múltiple

Regresión polinomial

Regre. mult. paso a paso

Correlación

Análisis varianza un lado

Análisis. vnza. sheffe

Analsis. vnaza. dos lados

Analsis. vnza. multilado

Anlsis. vnza, anidado

Anlsis. vnza. 2 nivel
SUBJECT ANALISIS

summary Chi-cuadrado

" " Test mediana de Fisher

$t$ tes Test Wilcoxon

regress Test Mann Whitney

" " Analisis Kruskal Wallis

" " Correlación Spearman

" "Analisis Friedman

" "Mediana y grado

correlat Test múltiple Neuman-Keul

anova Análisis regresión Probit

" "Integración numérica

" " Histograma y crosstabs

" "Scatter plot

" " Crosstabs múltiple

" " Reducción y clas.matriz data_tra

NOTA: Ver formato en help interactivo mediante campo subject!

Digite 1 para retornar al menu principal

DIGITO? :

PANTALLA 12

Al digitar 1 en la pantalla 12, STAT despliega la pantalla 1. 
8. (8) ANALISIS (STAT)

Al digitar 8 en la pantalla 1, STAT despliega la siguiente panta 11a:

OPCION: ANALISIS DE DATOS (STAT)

ENTER PARAMETER(S)

$\{$ IT MAY BE AS FOLLOWS: [/L), [/N] $\ldots . .([$ ] $=$ OPTIONAL $)\}$

PANTALLA 13

Esta pantalla está pidiendo los parámetros que determinarán el ámbito de los cálculos. Estos parămetros se colocan como un string de la siguiente forma

$$
/ \mathrm{P}_{1} / \mathrm{P}_{2} \cdots / \mathrm{P}_{\mathrm{n}}
$$

donde $\mathrm{P}_{1}, \mathrm{P}_{2} \ldots \mathrm{P}_{\mathrm{n}}$ son parámetros (todos opcionales) y que se detallan a continuación.

8.1. Uso de Parámetros.

8.1.1. Uso de /L.

Esta opción deja los resultados en un archivo generado por STAT, con el nombre STAT LIST.LST; no muestra los resultados por pantalla. Vuelve iterativamente a la pantalla 15.

8.1.2. Uso de $/ K$.

Esta opción indica que los análisis a efectuar se dan por archivo, para lo cual previamente se debe generar un archivo con la siguiente estructura 
NUMERO DE GRUPOS

NUMERO DE COLUMNAS

ANALISIS 1

ANALISIS 2

Cuando se activa este parámetro STAT consulta por el nombre de es te archivo y luego por el archivo de datos. Con esta opción se muestran los resultados por pantalla, pero no genera archivo de resultados. Vuelve a la pantalla 1 .

8.1.3. Uso de /N.

Esta opción permite ver los resultados por pantalla, pero no gene ra archivo de resultados. Vuelve iterativamente a la pantalla 15.

8.1.4. Uso de /P.

Esta opción indica a STAT que no numere las páginas del archivo de datos. Vuelve iterativamente a la pantalla 15.

8.1.5. Uso de /C.

Esta opción indica que los tîtulos (headings) de las columnas se entregan por archivos, para 10 cual previamente se debe generar un archivo con la siguiente estructura:

TITULO DE LA COLUMNA 1

TITULO DE LA COLUMNA 2 
Cuando se activa este parámetro, STAT consulta por el nombre de este archivo y luego por el archivo de datos. Vuelve iterativamente a la pantalla 15 .

8.2. Ingreso de Nombre de Archivos.

Después de haber ingresado los parámetros STAT despliega la siguiente pantalla

ENTER FILE(S): THE FIVE ORDER IS:

REQ-FILE (OPTIONAI)

HEAD-FILE (OPTIONAL)

DATA-FILL (NO OPTIONAL)

PANTALLA 14

en la cual pide el nombre de archivos en el siguiente orden:

(i) Archivos con títulos de columnas, si se ha activado el parámetro /C.

(ii) Archivo de análisis, si se ha activado el parámetro /K.

(iii) Nombre del archivo de datos (este es obligatorio)

8.3. Determinación de la matriz de datos.

Se puede trabajar con todo o parte del archivo de datos. Después de ingresar los nombres de los archivos, STAT consulta por el número de grupos (GROUPS?) y el número de columnas (COLUMNS?), determinando así la matriz de datos, para ambos casos se usan los siguientes formatos:

(i) $\mathrm{n}$ : indica que se consideraron en los análisis los n primeros grupos o columnas.

(ii) n,m: indica que se considerarán los m primeros grupos o columnas a partir de $n$, por ejemplo: si la consulta CoLUMNs? digitamos 4, 3; STAT considerará las columnas 4, 5 y 6 . 


\subsection{Análisis a realizar.}

Después de determinar la matriz de datos STAT despliega la siguiente pantalla (siempre que no se haya activado $/ \mathrm{K}$ )

MFANS: J, NOR $-2, F, M, L, U, D$

REGRESSION: $3, \mathrm{~K} 1, \mathrm{~K} 2,01,02$ or $4, \mathrm{~K} 1, \mathrm{~K} 2$ or $30, \mathrm{~K} 1$, IOPT

ANALYSIS NO? (use 45 for HELP)

PANTALLA 15

Después de solicitar el análisis con el formato adecuado STAT vuelve iterativamente a la pantalla 15, salvo que se indique un análisis 50 ó 0 (ver más adelante). En todos los análisis que se explican a continuación los parámetros marcados con $(<>)$ son opcionales.

\subsubsection{Listado de datos.}

Los análisis $-1,-2,-3$ generan distintos tipos de listados de los datos.

8.4.1.1. Uso de -1 .

E1 análisis -1 , genera un listado de los datos.

FORMATO: $-1,\langle$ FORM>, <LOWER>, 〈UPPER>, 〈DECIMAL 5>

FORM $=0$ : consulta por el formato de salida de los datos (en formato por cada columna)

FORM $=1$ : usa formato F6.1

FORM $=2$ : usa formato $\mathrm{F} 10.4$

FORM $=3$ : usa formato E12.4 (sólo vâlido con los análisis -2 y -3)

LOWER $=n$ : considera todas las columnas a partir de la n-ésima

UPPER $=\mathrm{m}$ : consider $\mathrm{a}$ desde $1 \mathrm{a}$ columna $\mathrm{n}$ hasta $1 \mathrm{a}$ columna $\mathrm{m}$

DECIMAL $5=\alpha$ : lista los datos con $\alpha$ lugares decimales, independientemente del formato utilizado en el parámetro FORM, siempre que $\alpha$ sea 
menor que el número de lugares decimales indicados en el formato.

Los valores por defecto son los siguientes

FORM : consulta por el formato de salida

LOWER : considera desde la primera columna

UPPER : considera hasta la última columna

DECIMAL 5 : considera el número de decimales indicados en formato.

8.4.1.2. Uso de -2 .

Genera un listado de datos en las mismas condiciones que -1 , pero agrega al final una estadística de los datos.

FORMATO: $-2,\langle$ FORM $>,\langle$ MISS $>,\langle$ LOWER $>,\langle$ UPPER $\rangle,\langle$ DECIMALS $\rangle$

Los parämetros se usan según se indicó en el apartado 8.4.1.1., para MISS usamos

MISS = $\quad-1$ : Iista las lineas que no contengan valores missing (los va lores missing se indican al general el archivo de datos). MISS = 1 lista todas las líneas, indicando los datos missing con con la palabra MISSING.

El valor por defecto es

MISS : considera MISS $=1 .-$

8.4.1.3. Uso de -3 .

Genera un listado de datos, sin la estadística final, y en las mismas condiciones que -2 .

FORMATO : $-3,\langle$ FORM $>,\langle$ MISS $>,\langle$ LOWER $>,\langle$ UPPER $>,\langle$ DECIMALS $>$

Los parámetros se usan según 10 indicado en los apartados 8.4.1.1. y 8.4 .1 .2 . 
8.4.2. Uso de 0 .

Permite determinar una nueva matriz de datos a partir del archivo de datos con que se está trabajando. Después de solicitar el análisis 0 , seguir el procedimiento que se indica en el apartado 8.3 .

8.4.3. Cálculo de medias y desviaciones típicas.

E1 análisis 1 permite realizar varios cálculos relacionados con la media y las desviaciones típicas.

$8 \cdot 4 \cdot 3.1$. Uso de 1 .

E1 análisis 1 calcula media, desviación típica, coeficiente de variación, valor mínimo, valor máximo para cada columna y además indica e1 número de valores missing en el set de datos.

\subsubsection{Uso de $1,-1$.}

El análisis $1,-1$, calcula lo mismo que el análisis 1 , pero no considera las filas que contengan valores missing.

8.4.3.2. Uso de $1, \mathrm{~K}$.

El análisis $1, K$ donde $K$ es un número de columna calcula $\Sigma x$ y $\sum \mathrm{x}^{2}$ para los datos de la columna $\mathrm{K}$.

8.4.3.3. Uso de $1, \mathrm{~K}_{1}, \mathrm{~K}_{2}$.

El análisis $1, K_{1}, K_{2}$ donde $K_{1} y K_{2}$ son dos números de columnas calcula $\sum x, \sum y, \sum x y, \sum(x-y), \sum x^{2}, \sum y^{2}, \sum(x-y)^{2}$ donde $x$ son los datos de la columna 1 , e y los datos de la columna 2 . 
8.4.3.4. Uso de $1, \mathrm{~K}_{1}, \mathrm{~K}_{2},-1$.

El anälisis $1, K_{1}, K_{2},-1$ hace lo mismo que el análisis $1, K_{1}$, $\mathrm{K}_{2}$, pero no considera las filas que contienen datos missing.

8.4.4. Uso de 2.

E1 análisis 2 realiza una prueba 5

FORMATO: $2, \mathrm{~K}_{1}, \mathrm{~K}_{2}, \mathrm{~N}$.

Los parámetros indican lo siguiente:

$\mathrm{K}_{1}=\mathrm{n} \quad$ : indica la columna $\mathrm{n}$

$\mathrm{K}_{2}=\mathrm{m} \quad$ : indica la columna $\mathrm{m}$

$\mathrm{N}=1$ : la prueba $t$ se realiza según la fórmula standard, en la cual se suponen varianzas agudas para cada columna.

$\mathrm{N}=2$ : la prueba 5 se realiza suponiendo que las varianzas no son iguales. Internamente realiza una prueba de igualdad de varianzas previa a 1a realización del análisis.

$\mathrm{N}=3$ : 1a prueba $\mathrm{t}$ se realiza considerando los datos como datos aparejados. En este caso se requiere que las columnas tengan el mismo número de datos.

8.4.5. Uso de 3 .

E1 análisis 3 determina los parámetros de la recta de regresión sus desviaciones típicas, además de la suma de cuadrados explicada y no explicada, el valor $t$ para la prueba de hipótesis.

FORMATO : $3, \mathrm{~K}_{1}, \mathrm{~K}_{2},<$ IOPT $1>,\langle$ IOPT $2>$

Los parámetros son Ios siguientes

$\mathrm{K}_{1}=\mathrm{n} \quad$ : indica la columna $\mathrm{n}$

$\mathrm{K}_{2}=\mathrm{m} \quad$ : indica $1 \mathrm{a}$ columna $\mathrm{m}$ 
IOPT $1=1 \quad$ : agrega un 1 istado con los valores de $1 \mathrm{a}$ columna m, calcu la según la recta de regresión con los datos de la colum na $\mathrm{n}$.

IOPT2 = 2 : consulta por valores especificos de la variable independiente (m) y entrega el valor de la variable dependiente (n). Este último valor es entregado como un intervalo de confianza.

Los valores por defecto son los siguientes

IOPT1 : no agrega listado de valores esperados para la variable de respuesta.

IOPT2 : no consulta por valores específicos de la variable independiente.

8.4.6. Uso de 4.

E1 análisis 4 entrega la recta de regresión de Bartlelts y los intervalos de confianza para la pendiente, además del valor 5 para 1 a prueba de hipótesis.

FORMATO : 4, KOLX, KOLY

Los parámetros son

$\mathrm{KOLX}=\mathrm{n}$ : indica que $\mathrm{n}$ es la columna que contiene los datos de la variable independiente.

KOLY $=\mathrm{m}$ : indica que $\mathrm{m}$ es $1 \mathrm{a}$ columna que contiene los datos de la variable dependiente.

8.4.7. Uso de 5.

E1 análisis 5 entrega una matriz de correlación de todas las columnas y una matriz de datos aparejados.

FORMATO : 5, <IOPT $>$ 
IOPT $=-1$ : calcula la matriz de conclusiones eliminando las filas que contienen datos missing.

8.4.8. Uso de 6.

El análisis 6 calcula una tabla de análisis de varianza (ANOVA) típica de un factor.

FORMATO : 6

8.4.9. Uso de 8 .

El análisis 8 calcula una tabla de análisis de varianza (ANOVA) de dos factores. No considera las filas con datos missing. FORMATO : 8

8.4.10. Uso de 9 .

E1 anâlisis 9 calcula la media y desviación típica de datos provenientes de poblaciones logarítmicas. Además entrega intervalos de confianza para la media.

FORMATO : 9

8.4.11. Uso de 11 .

E1 análisis 11, realiza una prueba chi-cuadrado para datos conjugados por terminal, en contraste con los otros análisis, éste pide que los datos se ingresen en el momento de realizar el análisis.

FORMATO : 11, NCOL, NROW

NCOL $=n$ : indica que la matriz de datos tiene $n$ columnas.

NROW $=m$ : indica que $1 \mathrm{a}$ matriz de datos tiene $\mathrm{m}$ filas. 
8.4.12. Uso de 12.

E1 análisis 12, realiza el test de la mediana, usando Fisher - chi-cuadrado sobre muestras independientes. Fisher se usa si $N<40$ ó si alguna frecuencia es menor que 5 , en caso contrario usa chi-cuadra do.

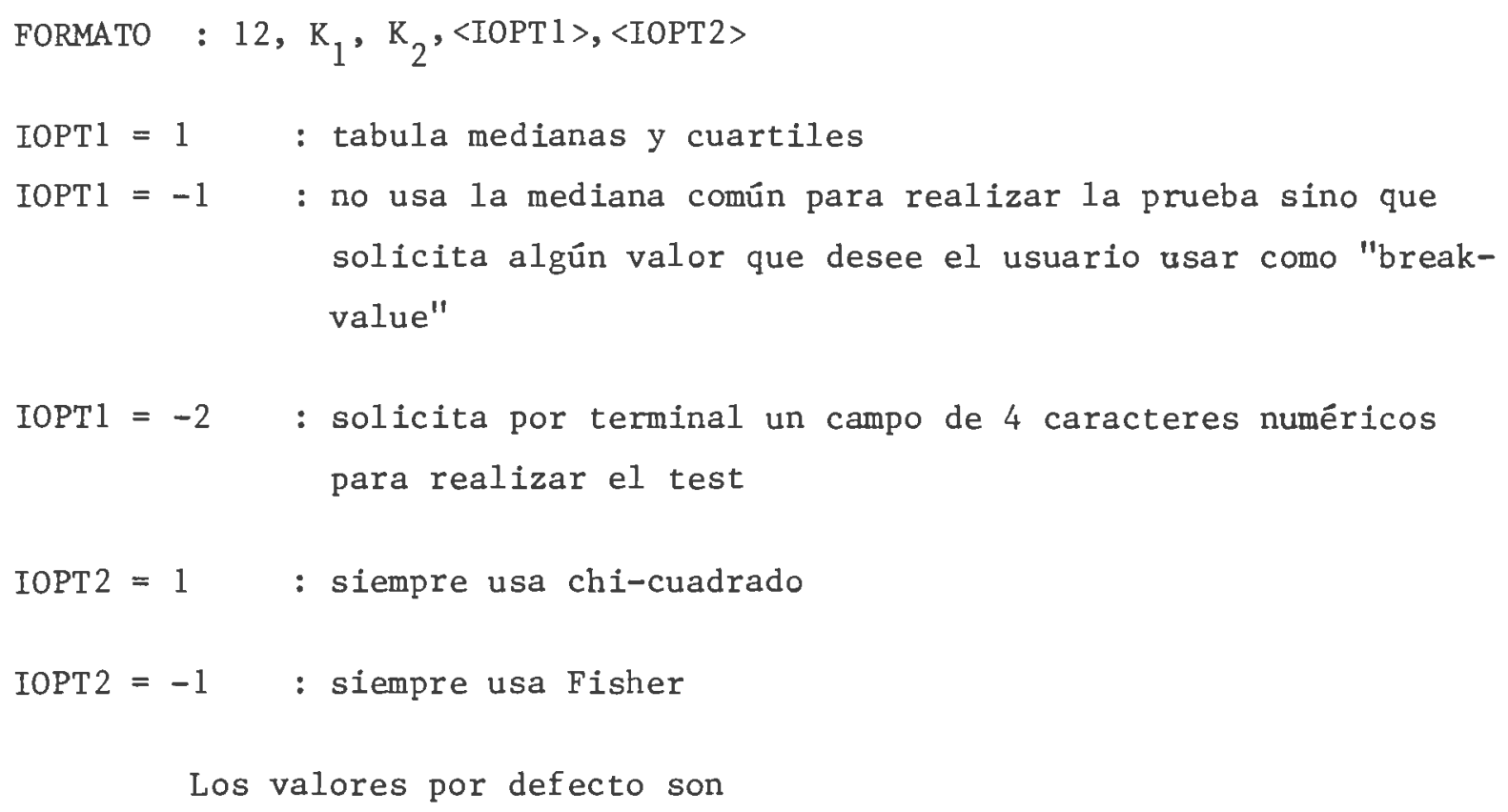

E1 análisis 13 realiza el test de Welcoxon, que es el equivalente no paramétrico del test $t$ de datos aparejados.

FORMATO : $13, \mathrm{~K}_{1}, \mathrm{~K}_{2}$. 
8.4.14. Uso de I4.

El análisis 14 realiza el test de Mann-Whitney, que es el equivalente no paramétrico del test $t$ de datos no aparejados.

FORMATO $14, \mathrm{~K}_{1}, \mathrm{~K}_{2}$.

8.4.15. Uso de 15.

E1 análisis 15, realiza el test de Kruskol-Wallis que es el equi valente no paramétrico del ANOVA de un factor.

FORMATO : 15, <IOPT>

IOPT $=-1$ : elimina las filas con datos missing y agrega una tabla de diferencias.

8.4.16. Uso de 16 .

El análisis 16 realiza el test de correlación de rangos de Spearman.

FORMATO : $16, \mathrm{~K}_{1}, \mathrm{~K}_{2}$.

8.4.17. Uso de 17 .

El análisis 17 realiza el análisis de Friedman, que es el equivalente no paramétrico del ANOVA de dos factores.

FORMATO : 17

8.4.18. Uso de 18.

E1 análisis 18, realiza integración numérica.

FORMATO : $18, \mathrm{~K}_{1}, \mathrm{~K}_{2},<\mathrm{ARG} 1>,<\mathrm{ARG} 2>$ 
$\mathrm{K}_{1}=\mathrm{n}$ : indica que la columna $\mathrm{n}$ será usada como los valores de $\mathrm{x}$. $\mathrm{K}_{2}=\mathrm{m}$ : indica que la columna m será usada como los valores de $\mathrm{y}$. ARG1 = 0 : entrega sólo el área final. ARG2 = 1 : además entrega una tabla de áreas parciales ARG3 = 1 : calcula el área por el método de trapecios modificados, des pues de un "break-valur" solicitado por terminal.

Los valores por defecto son

ARG1 : entrega sólo el área final

ARG2 : no pide "break-value"

8.4.19. Uso de 19.

El análisis 19, calcula mediana para cada columna y ordena las columnas (rank).

FORMATO : 19, <IOPT>

IOPT $=0$ : calcula las medianas para todas las columnas, sin considerar los datos missing.

$\mathrm{IOPT}=\mathrm{n}:$ ordena $1 \mathrm{a}$ columna $($ rank) $\mathrm{n}$.

El valor por defecto es

IOPT $\quad:$ IOPT $=0$

8.4.20. Uso de 20.

E1 análisis 20, realiza una tabulación cruzada o un histograma.

FORMATO : $20, \mathrm{~K}_{1}, \mathrm{~K}_{2},\langle\mathrm{NINT}\rangle$

$\mathrm{K}_{1} \neq \mathrm{K}_{2} \quad$ : hace la tabulación cruzada entre las columnas $\mathrm{K}_{1}$ y $\mathrm{K}_{2}$ 
$\mathrm{K}_{1}=\mathrm{K}_{2}$ : hace el histograma para la columna $\mathrm{K}_{1}$

NINT $=\mathrm{k}$ : usa $\mathrm{k}$ intervalos tanto para la tabulación cruzada como para el histograma, $(3 \leqslant k \leqslant 10)$

E1 valor por defecto es

NINT $\quad:$ NINT $=5$

8.4.21. Uso de 21 .

E1 análisis 21, realiza gráficos de una variable contra otra.

FORMATO: $21, \mathrm{~K}_{1}, \mathrm{~K}_{2},\langle\mathrm{IOPT} 1>,\langle\mathrm{IOPT} 2\rangle,\langle\mathrm{IOPT} 3\rangle$

$\mathrm{K}_{1}=\mathrm{n}$ : los datos de la columna $\mathrm{n}$ se usan como eje horizontal. Este eje se divide en 50 partes.

$\mathrm{K}_{2}=\mathrm{m}$ : los datos de la columna $\mathrm{m}$, se usan como eje vertical. Este eje puede dividirse desde 20 hasta 50 partes.

IOPT 1 = $\mathrm{k}$ : divide en $\mathrm{k}$ partes los datos de la columna $\mathrm{K}_{2}$ (eje vertical) $10 \leqslant k \leqslant 50$.

IOPT2 = 1: permite cambiar de ubicación el punto de mayor coordenadas, pro duciendo un reordenamiento del gráfico.

IOPT $3=-1$ : permite trabajar con una escala logarítmica en el eje vertical.

Los valores por defecto son:

IOPT1 : divide los datos de la columna $\mathrm{K}_{2}$ (eje vertical) en 25 partes.

IOPT2 : ubica la coordenada mayor en la parte superior del gráfico.

IOPT3 : no usa escala logarítmica. 
8.4.22. Uso de 22.

El análisis 22 permite cambiar los datos en el archivo de trabajo temporalmente, los datos originales permanecen invariables.

FORMATOS : (1) $22, \mathrm{~K}_{1}$

$\mathrm{K}_{1}=\mathrm{n}$ : la columna $\mathrm{n}$ es ordenada en forma ascendente. Los valores miss ing se ponen al final de la lista

(2) $22,-K_{1}$

$\mathrm{K}_{1}=\mathrm{n} \quad:$ la columna $\mathrm{n}$ es ordenada en forma descendente. Los valores miss ing se ponen al final de la lista

(3) $22, \mathrm{NK}, \mathrm{K}_{1},-1$

$\mathrm{NK}=\mathrm{n} \quad$ : indica el número $\mathrm{n}$ total de columna del archivo original.

$\mathrm{K}_{1}=\mathrm{m}$ : reduce el número $\mathrm{n}$ total de columnas a m columnas

(4) $22, \mathrm{~K}_{1}, \mathrm{~K}_{2}, \mathrm{CODE}$

KOL1 $=\mathrm{n}$ : 1a columna $\mathrm{n}$ es reemplazada por la columna $\mathrm{m}$ (ingresada por KOL2 = m) según 10 indicado por CODE.

KOL $2=m$ : los datos de la columna $\mathrm{m}$, se ponen en la columna $\mathrm{n}$ (ingresada por KOLl $=n$ ) según lo indicado por CODE.

KOL2 $=-1$ : en este caso se ignora 1 a columna $n$ (ingresada por KOL $1=n$ ) y el cambio se hace en todas las columnas.

COTE $=\mathrm{k}$ : produce el cambio indicado por $\mathrm{k}$ según la lista siguiente:

1 raíz cuadrada

2 exponencial

3 logaritmo natural

4 logaritmo decimal

5 cuadrado

6 igual (copia la columna $n$ en la columna m) 


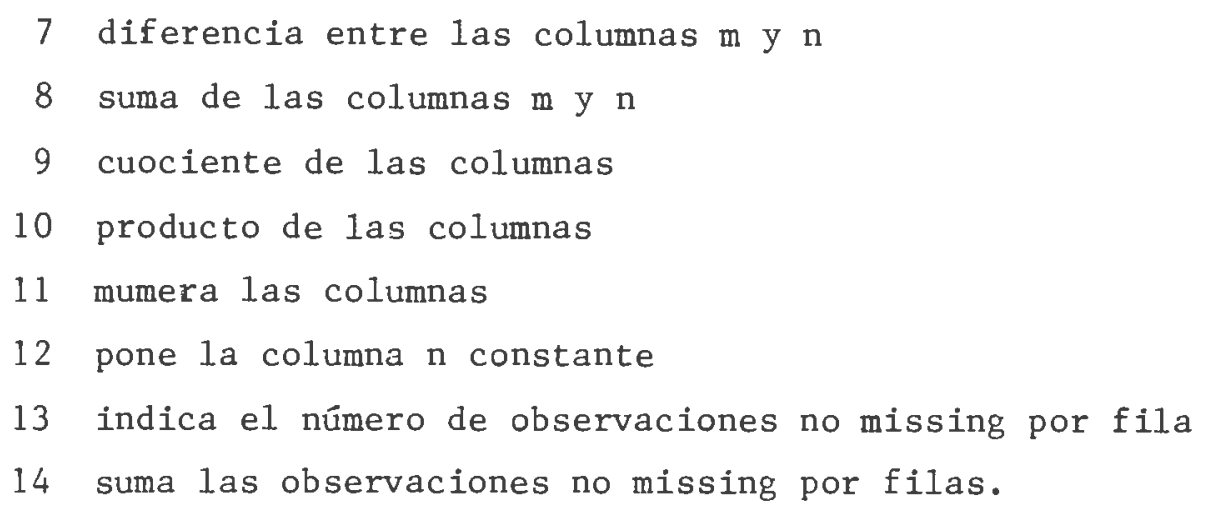

8.4.23. Uso de 23.

E1 análisis 23 produce un intercambio de filas y columnas.

FORMATO : 23

8.4.24. Uso de 30.

E1 análisis 30 realiza una regresión múltiple, usando todos los datos en la matriz de trabajo.

FORMATO : $30, \mathrm{~K}\langle$ IOPT $>$

$\mathrm{K}=\mathrm{n}$ : indica que la columna $\mathrm{n}$ es la variable de respuesta IOPT $=\mathrm{k}$ : donde $\mathrm{k}>0$, imprime la tabla de residuos.

Valor por defecto

IOPT : no imprime la tabla de residuos.

8.4.25. Uso de 31.

E1 análisis 31, realiza un análisis de varianza de dos factores y para varios niveles.

FORMATO ; $31, \mathrm{n}$

7 . intica e1 número de niveles. 
8.4.26. Uso de 32 .

E1 análisis 32 realiza una regresión polinomial.

FORMATO : $32, \mathrm{NX}, \mathrm{NY}, \mathrm{MAX},\langle\mathrm{IOPT}>$

$\mathrm{NX}=\mathrm{n} \quad$ : indica que $1 \mathrm{a}$ columna $\mathrm{n}$ es considerada como la variable dependiente.

$N Y=m$ : indica que $1 \mathrm{a}$ variable $\mathrm{m}$ es considerada como la variable depen diente.

$\operatorname{MAX}=k: 1 \leqslant n \leqslant 4$, indica el grado del polinomio de regresión.

IOPT $=\ell: \ell \neq 0$, imprime al final una table de residuos.

E1 valor por defecto es:

IOPT : no imprime la tabla de residuos.

8.4.27. Uso de 34.

E1 análisis 34, realiza una regresión múltiple paso a paso (STEPWISE).

FORMATO : $34,\langle$ IOPT $1>,<$ IOPT 2>

IOPT $1=\mathrm{n} \quad: \mathrm{n} \neq 0$, genera tabla de residuos.

IOPT2 $=\mathrm{m} \quad: \mathrm{m} \neq 0$, consulta por un valor de la varianza explicada bajo la cual no continúa entrando variables forzadas.

Después de introducir el formato, consulta por un string de nk caracteres, ( $\mathrm{nk}=$ número de columnas), con los siguientes códigos:

Veriable independiente forzada a entrar en el análisis.

2 Variable que no debe considerarse en el análisis.

3 Variable dependiente. 
Los valores por defecto son:

IOPT1 : no genera tabla de residuos.

IOPT2 : el valor que asume es 00 (es decir entran todas las variables).

8.4.27. Uso de 46.

E1 análisis 46, permite cambiar las probabilidades con que trabaja STAT para los análisis.

8.4.28. Uso de 47.

E1 análisis 47 permite cambiar el valor para datos missing.

8.4.29. Uso de 48.

El análisis 48 permite hacer comentarios (escribir textos) al final del listado de resultados.

8.4.30. Uso de 50.

E1 análisis 50 produce la salida a la pantalla 1.

8.4.31. Uso de 70.

E1 análisis 70 permite leer un nuevo archivo de trabajo, tal co mo se pide en 1a pantalla 14. (ver página 159). 
9. (9) DATOS DESDE UN EMISOR (OUTCHK)

Al digitar 9, aparece la siguiente pantalla

OPCION: DATOS DESDE UN EDITOR (OUTCHK)

La entrada de datos (OUTLAY) puede ser proporcionada por un archivo creado por un editor. E1 programa OUTCHK verifica la corrección de los datos que han sido creados bajo esta modalidad.

La salida proporcionada por este programa es un listado

de todas las líneas que presentan errores.

Los datos que deben ser dados al programa son

los siguientes (y en este orden):

Indicar opcion 1

SWITCHES

NOMBRE ARCHIVO A ANALIZAR

NUMERO DE COLUMNAS

FORMATO ( $\mathrm{si}$ coloca /f )

SWITCHES :

$$
\begin{aligned}
/ A= & \text { Liste todas las líneas (asume listar solo } \\
& \text { líneas incorrectas) } \\
/ L= & \text { Salida por impresora (implica } / A \text { ) } \\
/ f= & \text { Formato para lectura del archivo (asume } \\
& 15 \mathrm{~F} 11.0 \text { ) }
\end{aligned}
$$

INDIQUE OPCION :

(1) Análisis archivo

(99) Retornar menu principal

OPCION :

PANTALLA 16

9.1. Uso de 1.

Al digitar 1 en la pantalla 16, STAT consulta por SWITCHES, VOMBKE DE ARCHIVO A ANALIZAR, NUMERO DE COLUMNAS y FORMATO (sólo si se tia usado el switch /f) un parámetro en cada línea. Los switches son 
$\begin{array}{ll}\text { /A } & \text { : lista todas las líneas } \\ \text { /L } & : \text { salida por impresora (cuando se ha usado /A) } \\ \text { /f } & \text { : formato de lectura por archivo }\end{array}$

Los valores por defecto son:

/A : solo listas líneas incorrectas

/I. $\quad$ : muestra los resultados por pantalla

/f : usa el formato $15 F 11.0$

9.2. Uso de 99.

Al digitar 99 en la pantalla 16, vuelve a la pantalla 1.

10. (10) ANALISIS ESPECIALES (TABELL)

Al digitar 10 en la pantalla 1, STAT despliega la siguiente panta11a

OPCION: ANALISIS ESPECIALES (TABELL)

Este programa es usada en lugar de una tabla estadística. Permite conocer la probabilidad

de un valor obtenido por un test estadístico.

La entrada al programa es la siguiente:

$! \mathrm{P}, \mathrm{N} 1, \mathrm{~N} 2$

En que ! = indica el tipo de tabla a usar puede ser:

$$
\begin{gathered}
\text { T para T-valor } \\
\text { F para F-valor } \\
\text { X para CHI-2 (probabilidad) } \\
\text { Z para valor normal } \\
\text { C cálculo de chi-2 (Ccolumna,fila) } \\
\text { E FIN DEL PROGRAMA } \\
\text { P = es 1a probabilidad } \\
\text { N1 y N2 = son los grados de libertad (N2 sólo } \\
\text { para F, N1 no usado para Z) }
\end{gathered}
$$

INDIQUE OPCION :

(1) análisis (TABELL)

(99) retorno menu principal

OPCION : 
10.1. Uso de 1.

Al digitar 1 en la pantalla 17, STAT consulta la probabilidad de seada, según se explica en la pantalla 17.

10.2. Uso de 99.

Al digitar 99 en la pantalla 17, STAT vuelve a la pantalla 1.

11. (99) FIN.

Al digitar 99 en la pantalla l, STAT libera el terminal y lo de ja a disposición del usuario.

12. RECOMENDACIONES PARA EL USUARIO.

La forma más cómoda para trabajar con STAT es mandar a digitar los datos a CECUN, indicando el nombre del archivo. Este archivo puede ser digitado en formato libre, pues usando la opción 4 de STAT, se deja en formato OUTLAY, con la cual trabaja STAT. Para realizar análisis so bre los datos, sólo para ver en pantalla use la siguiente secuencia de instrucciones:

\$ STAT 11

8

$/ \mathrm{N}$

<NOMBRE DEL ARCHIVO DE DATOS>

ANALISIS 1

ANALISIS 2

ANALISTS $\mathrm{N}$

o (fin del análisis)

99 (salida de STAT) 
Si desea generar archivo de resultados, use la siguiente secuen cia de instrucciones:

\$ STAT 11

8

$/ \mathrm{L}$

<NOMBRE DEL ARCHIVO DE DATOS>

ANALISIS 1

ANALISIS 1

$\vdots$

ANALISIS $\mathrm{N}$

50 (fin de análisis)

99 (salida de STAT)

Ahora los resultados están disponibles en un archivo STAT LIST. LST. 